\title{
Pricing of Two Kinds of Power Options under Fractional Brownian Motion, Stochastic Rate, and Jump-Diffusion Models
}

\author{
Kaili Xiang, Yindong Zhang, and Xiaotong Mao \\ School of Economic Mathematics, Southwestern University of Finance and Economics, Chengdu 610074, China \\ Correspondence should be addressed to Kaili Xiang; xiangkl@swufe.edu.cn
}

Received 27 March 2014; Accepted 31 August 2014; Published 21 October 2014

Academic Editor: Fasma Diele

Copyright (C) 2014 Kaili Xiang et al. This is an open access article distributed under the Creative Commons Attribution License, which permits unrestricted use, distribution, and reproduction in any medium, provided the original work is properly cited.

\begin{abstract}
Option pricing is always one of the critical issues in financial mathematics and economics. Brownian motion is the basic hypothesis of option pricing model, which questions the fractional property of stock price. In this paper, under the assumption that the exchange rate follows the extended Vasicek model, we obtain the closed form of the pricing formulas for two kinds of power options under fractional Brownian Motion (FBM) jump-diffusion models.
\end{abstract}

\section{Introduction}

The pricing of exotic options is often an optimal stochastic problem, in which the stochastic process sometimes follows jump-diffusion process. The issue gets more complicated because the distribution of the maximum is difficult to derive. There is no doubt that we should use jump-diffusion process to describe the changing behaviors of financial markets, instead of diffusion process, but the pricing process is more complex. Market quotations, trade practices, and information dissemination are three important factors which effect trade speed and transaction volume. Therefore, a reasonable pricing is the premise which will active the exchange market.

In recent years, with the exception of European and American options, a large number of new financial derivatives are derived in the international financial derivative market. Among them, the power option is one of the new typical options. The research of the power options has significant meanings both theoretical and practical. The BS [1] model has become an indispensable tool for option pricing and hedging in the finance industry. However, it is well documented that the Geometric Brownian Motion (GBM) assumption for the underlying asset's price dynamics in the BS model fails to reflect the real facts: market return data display excess kurtosis (peaked and fat-tailed distributions), skewness, volatility clustering, long-range dependence and large, sudden movements, and so forth. These observations reveal that a simple GBM assumption misses some important features of the data. Therefore, many different option valuation models with realistic price dynamics have been currently proposed and tested. Some of these models include jump-diffusion model, subordinated processes, pure jump processes, Lévy processes, stochastic volatility model, regime-switching model, GARCH model, processes driven by FBM, and others. Except for FBM, these models give rise to incomplete market. Hu and Oksendal [2] and Elliott and van der Hoek [3] have proved that there is no arbitrage in the FBM market if the wick product is used in the definition of stochastic integration. Compared with the traditional efficient market theory, use the fractional market theory to describe that the movement of the actual market is more appropriate and accurate. Pricing options with stochastic interest rate under jump-diffusion models are an important field in recent years.

In the FBM market, very little work on the options valuation is considered. $\mathrm{Hu}$ and Oksendal [2] have derived a formula for the price at time $t=0$ of a European option. Necula [4] has extended the formula in [2] for every time $t \in[0, T]$. Liu and Yang $[5,6]$ considered the European contingent claim and compound option for the ease of a nonconstant but deterministic volatility using the quasiconditional expectation. Xue and Wang [7] also consider the pricing of the extremum options for the two risky assets type. Elliott and Chan [8] have obtained a closed-form solution for perpetual American options whose maturity goes to 
infinity by applying quadratic approximation. Peng [9] has also obtained an explicit price of perpetual American put for a fractional O-U model. Deng and Lin [10] have considered approximate valuation for the American put option with finite maturity date using compound option approach.

In this paper, under the assumption that the exchange rate obeys the expanding Vasicek models, we obtain the pricing formulas of two kinds of power options under fractional jump-diffusion models.

\section{The Fundamental Theorem and Lemma}

In this section, we state several theorems and lemmas presented in [11].

Theorem 1. Let $X \sim N(0,1), Y \sim N(0,1), \operatorname{Cov}(X, Y)=\rho$. Then, we have

$$
\begin{aligned}
E\left[\exp (c X+d Y) \chi_{a X+b Y \geq k}\right] & \\
= & \exp \left(\frac{1}{2}\left(c^{2}+d^{2}+2 \rho c d\right)\right) \\
& \times N\left(\frac{a c+b d+\rho(a d+b c)-k}{\sqrt{a^{2}+b^{2}+2 \rho a b}}\right),
\end{aligned}
$$

where $a, b, c, d, k \in R$.

Lemma 2. Let $X \sim N\left(0, \sigma_{X}^{2}\right), Y \sim N\left(0, \sigma_{Y}^{2}\right), \operatorname{Cov}(X, Y)=\rho$. Then, we have

$$
\begin{aligned}
& E\left[\exp (c X+d Y) \chi_{a X+b Y \geq k}\right] \\
& \quad=\exp \left(\frac{1}{2}\left(c^{2} \sigma_{X}^{2}+d^{2} \sigma_{Y}^{2}+2 \rho c d \sigma_{X} \sigma_{Y}\right)\right) N(D),
\end{aligned}
$$

where $a, b, c, d, k \in R$ and

$$
D=\frac{a c \sigma_{X}^{2}+b d \sigma_{Y}^{2}+\rho(a d+b c) \sigma_{X} \sigma_{Y}-k}{\sqrt{a^{2} \sigma_{X}^{2}+b^{2} \sigma_{Y}^{2}+2 \rho a b \sigma_{X} \sigma_{Y}}} .
$$

Definition 3. We have some assumptions as follows:

$$
\begin{aligned}
& F(w)=\sum_{\alpha \in I} a_{\alpha} H_{\alpha}(w) \in(S)_{H}^{*}, \\
& G(w)=\sum_{\beta \in I} b_{\beta} H_{\beta}(w) \in(S)_{H}^{*} .
\end{aligned}
$$

We define wick integral as follows:

$$
F \diamond G(w)=\sum_{\alpha, \beta \in I} a_{\alpha} b_{\beta} H_{\alpha+\beta}(w) .
$$

Definition 4. We define the function $Y: R \rightarrow(S)_{H}^{*}$, s.t. $Y(t) \diamond$ $W_{H}(t)$ is integrated in $(S)_{H}^{*}$. Then we can define the stochastic integration of $Y$ about $\left\{B_{H}(t), t \in R, H \in(0,1)\right\}$ as follows:

$$
\int_{R} Y(t) d B_{H}(t)=\int_{R} Y(t) \diamond W_{H}(t) d t,
$$

where $W_{H}(t)$ means fractional noise and $\diamond$ means wick integral.

\section{Our Models}

3.1. Interest Rate Model. We assume that the interest rate follows the extended Vasicek model in this paper. That means it has the following form:

$$
d r(t)=(a(t)-b(t) r(t)) d t+\sigma_{r}(t) d B^{Q}(t),
$$

where $a(t)$ affects long-term average interest rate; $b(t)$ is the average recovery rate which adjusts the relationship of shortterm and long-term; and $\sigma_{r}(t)$ is fluctuation ratio.

As we all know, if we want to find out the discounted factor, firstly we need to find out the discount rate $\int_{t}^{T} r(s) d s$. By the Itô theorem, we have

$$
d\left(e^{n(s)} r(s)\right)=e^{n(s)}\left(a(s) d s+\sigma_{r}(s) d B^{Q}(s)\right),
$$

where $n(s)=\int_{0}^{s} b(u) d u$. Then by the integral both sides of the equation above on $[t, s]$, we have

$$
\begin{aligned}
r(s)= & r(t) e^{n(t)-n(s)}+\int_{t}^{s} e^{n(t)-n(s)} a(u) d u \\
& +\int_{t}^{s} e^{n(t)-n(s)} \sigma_{r}(u) d B^{Q}(u) .
\end{aligned}
$$

Further,

$$
\begin{aligned}
\int_{t}^{T} r(s) d s & \\
= & r(t) \int_{t}^{T} e^{n(t)-n(s)} d s+\int_{t}^{T} \int_{t}^{s} e^{n(t)-n(s)} a(u) d u d s \\
& +\int_{t}^{T} \int_{t}^{s} e^{n(t)-n(s)} \sigma_{r}(u) d B^{Q}(u) \\
= & r(t) \int_{t}^{T} e^{n(t)-n(s)} d s+\int_{t}^{T} a(u) \int_{t}^{s} e^{n(t)-n(s)} d s d u \\
& +\int_{t}^{T} \sigma_{r}(u) \int_{t}^{s} e^{n(t)-n(s)} d s d B^{Q}(u) \\
= & +(t) m(t, T)+\int_{t}^{T} a(u) m(u, T) d u \\
& +\int_{t}^{T} \sigma_{r}(u) m(u, T) d B^{Q}(u) \\
= & G\left(t, T, r_{t}\right)+\int_{t}^{T} \sigma_{r}(u) m(u, T) d B^{Q}(u),
\end{aligned}
$$

where

$$
\begin{gathered}
m(u, v)=\int_{u}^{v} e^{n(t)-n(s)} d s \\
G\left(t, T, r_{t}\right)=r(t) m(t, T)+\int_{t}^{T} a(u) m(u, T) d u .
\end{gathered}
$$


3.2. Jump-Diffusion Model. We assume the market is a full market with continuous time, and there exist two kinds of continuous trading assets, one of which is risk-free bonds. The price process of bonds $M(t)$ satisfies the following equation:

$$
\frac{d M(t)}{M(t)}=r(t) d t, \quad M(0)=1,
$$

where $r(t)$ is a continuous function of time $t$. The other trading asset is the risk asset $S(t)$; the price process of $S(t)$ satisfies the following equation:

$$
d S(t)=S(t)\left[\mu(t) d t+\sigma(t) d B(t)+\left(e^{J(t)}-1\right) d Q_{t}\right],
$$

where $B(t)$ is Brownian motion; $Q_{t}$ is the number of random jumps of underlying asset price within $[0, t]$ and it follows the Poisson process with the parameter $\lambda ; J(t)$ is the random variable which follows the normal distribution $N\left(-\sigma_{J}^{2} / 2, \sigma_{J}^{2}\right)$; $e^{J(t)}-1$ is the height of the stock price jump; $\mu(t)$ is the expected rate of return; and $\sigma^{2}(t)$ is the volatility.

Solving (14), we have

$$
\begin{aligned}
S(t)=S_{0} \exp \left[\int_{0}^{t}\left(\mu(t)-\frac{\sigma^{2}(t)}{2}\right) d s+\int_{0}^{t} \sigma(t) d B(t)\right. \\
\left.+\sum_{i=1}^{Q_{t}} J(i)\right] .
\end{aligned}
$$

In addition, jump-diffusion model is independent of the risk appetite, so risk-free interest rate $r(t)$ can be used to take place of the expected rate of return $\mu(t)$ according to the riskneutral valuation principles. Consequently, the formula (15) can be rewritten as follows:

$$
\begin{aligned}
S(t)=S_{0} \exp \left[\int_{0}^{t}\left(r(t)-\frac{\sigma^{2}(t)}{2}\right) d s+\int_{0}^{t} \sigma(t) d B(t)\right. \\
\left.+\sum_{i=1}^{Q_{t}} J(i)\right] .
\end{aligned}
$$

3.3. Fractional Jump-Diffusion Model. Fractional jumpdiffusion model is the extension of jump-diffusion model because of the replacement of Brownian motion $B(t)$ with fractional Brownian motion $B_{H}(t)$ in the model. The risk-neutral dynamics of the underlying asset is given as

$$
d S(t)=S(t)\left[(r(t)-\lambda \theta) d t+\sigma(t) d B_{H}(t)+J(t) d Q_{t}\right]
$$

where $\left\{B_{H}(t), 0 \leq t \leq T\right\}$ is the fractional Brownian motion in probability space $(\Omega, F, P) ; Q_{t}$ is the number of random jumps of underlying asset price within $[t, T]$, which follows Poisson process with the parameter $\lambda$; $J(t)$ is the height of the jump on stock price, satisfying $\ln (1+J(t)) \sim N\left(\ln (1+\theta)-\left(\sigma^{2} / 2\right), \sigma^{2}\right)$; $r(t)$ is the expected rate of return; and $\sigma^{2}(t)$ is the volatility.

From (17), we have

$$
\frac{d S(t)}{S(t)}=(r(t)-\lambda \theta) d t+\sigma(t) d B_{H}(t)+J(t) d Q_{t} .
$$

According to the definitions and properties of stochastic calculus and function $\exp \diamond(X)$, we get

$$
\begin{aligned}
S(t)= & S_{0} \exp \diamond\left[\int_{0}^{t}(r(s)-\lambda \theta) d s\right. \\
& \left.\quad+\int_{0}^{t} \sigma(s) d B_{H}(s)+\sum_{i=1}^{Q_{t}} \ln (1+J(i))\right] \\
= & S_{0} \prod_{i=1}^{Q_{t}}(1+J(i)) \\
& \times \exp \diamond\left[\int_{0}^{t}(r(s)-\lambda \theta) d s+\int_{0}^{t} \sigma(s) d B_{H}(s)\right] \\
= & S_{0} \prod_{i=1}^{Q_{t}}(1+J(i)) \\
& \times \exp \left[\int_{0}^{t}\left(r(s)-\lambda \theta-H \sigma^{2}(s) s^{2 H-1}\right) d s\right. \\
& \left.+\int_{0}^{t} \sigma(s) d B_{H}(s)\right] .
\end{aligned}
$$

\section{Pricing of Two Kinds of Power Options under FBM, Stochastic Rate, and Jump-Diffusion Models}

In this section, we obtain pricing formulas of two kinds of power options.

4.1. Pricing of the First Kind of Power Option. From (11), we have

$$
\int_{t}^{T} r(s) d s=G\left(t, T, r_{t}\right)+\int_{t}^{T} \sigma_{r}(u) m(u, T) d B^{Q}(u),
$$

where $m(u, v), G\left(t, T, r_{t}\right)$ are given by (12), (23), respectively.

Suppose that underlying assets jump $m$ times during $[t, T]$, then

$$
\begin{aligned}
S_{T}=S_{t} \prod_{i=1}^{m}(1+J(i)) \\
\times \exp \left[\int_{t}^{T}\left(r(s)-\lambda \theta-H \sigma^{2}(s) s^{2 H-1}\right) d s\right. \\
\left.+\int_{t}^{T} \sigma(s) d B_{H}(s)\right] .
\end{aligned}
$$

Theorem 5. The formula of the first kind of call power option with exercise price $K$ at exercise date $T$ is

$$
\begin{aligned}
C & \left(t, r_{t}, S_{t}, K\right) \\
& =\sum_{m=0}^{\infty}\left[\exp (-\lambda(T-t)) \frac{(\lambda(T-t))^{m}}{m !}\left(I_{c}-I I_{c}\right)\right],
\end{aligned}
$$


4

Abstract and Applied Analysis

where

$$
\begin{aligned}
& I_{c}=S_{t}^{n} \prod_{i=1}^{m}(1+j(i)) \exp \left(s_{1}\right) \cdot N\left(d_{1}\right) \text {, } \\
& I I_{c}=K \exp \left[\frac{\sigma_{X}^{2}}{2}-G\left(t, T, r_{t}\right)\right] \cdot N\left(d_{2}\right), \\
& s_{1}=\frac{(n-1)^{2}}{2} \sigma_{X}^{2}+\frac{n^{2}-n}{2} \sigma_{Y}^{2}+(n-1) G\left(t, T, r_{t}\right) \\
& -n \int_{t}^{T} \lambda \theta d s, \\
& d_{1}=\left(\ln \left[S_{t} \cdot \sqrt[n]{\frac{\prod_{i=1}^{m}(1+j(i))}{K}}\right]+G\left(t, T, r_{t}\right)-\int_{t}^{T} \lambda \theta d s\right. \\
& \left.+(n-1) \sigma_{X}^{2}+\frac{2 n-1}{2} \sigma_{Y}^{2}\right)\left(\sqrt{\sigma_{X}^{2}+\sigma_{Y}^{2}}\right)^{-1}, \\
& d_{2}=\left(\ln \left[S_{t} \cdot \sqrt[n]{\frac{\prod_{i=1}^{m}(1+j(i))}{K}}\right]+G\left(t, T, r_{t}\right)\right. \\
& \left.-\int_{t}^{T} \lambda \theta d s-\sigma_{X}^{2}-\frac{\sigma_{Y}^{2}}{2}\right)\left(\sqrt{\sigma_{X}^{2}+\sigma_{Y}^{2}}\right)^{-1} .
\end{aligned}
$$

Proof. According to the No-Arbitrage pricing theory, we get

$$
\begin{aligned}
C\left(t, r_{t}, S_{t}, K\right) \\
\quad=E\left[\exp \left(-\int_{t}^{T} r(s) d s\right)\left(S_{T}^{n}-K\right) \chi_{S_{T}^{n}>K}\right] \\
=\sum_{m=0}^{\infty}\left[\exp (-\lambda(T-t)) \frac{(\lambda(T-t))^{m}}{m !} I_{c}-I I_{c}\right] .
\end{aligned}
$$

Let

$$
\begin{gathered}
X=\int_{t}^{T} \sigma_{r}(u) m(u, T) d B(u), \\
Y=\int_{t}^{T} \sigma(s) d B_{H}(s),
\end{gathered}
$$

then

$$
\begin{gathered}
X \sim N\left(0, \int_{t}^{T} \sigma_{r}(u) m(u, T) d u\right)=N\left(0, \sigma_{X}^{2}\right), \\
Y \sim\left(0,2 H \int_{t}^{T} \sigma^{2}(u) u^{2 H-1} d u=N\left(0, \sigma_{Y}^{2}\right)\right) .
\end{gathered}
$$

Then we have

$$
I_{c}=E\left[\exp \left(-\int_{t}^{T} r(s) d s\right) S_{T}^{n} \chi_{S_{T}^{n} \geq K}\right] .
$$

Since

$$
\begin{aligned}
& \exp \left[-\int_{t}^{T} r(s) d s\right] S_{T}^{n} \\
& =\exp \left[-\int_{t}^{T} r(s) d s\right] S_{t}^{n} \prod_{i=1}^{m}(1+j(i)) \\
& \cdot \exp \left[n \int_{t}^{T}\left(r(s)-\lambda \theta-H \sigma^{2}(s) s^{2 H-1}\right) d s\right. \\
& \left.+n \int_{t}^{T} \sigma(s) d B_{H}(s)\right] \\
& =S_{t}^{n} \prod_{i=1}^{m}(1+j(i)) \\
& \times \exp \left[(n-1) \int_{t}^{T} r(s) d s\right. \\
& \left.+n \int_{t}^{T}(-\lambda \theta) d s-\frac{n}{2} \sigma_{Y}^{2}\right] \cdot e^{n Y} \\
& =S_{t}^{n} \prod_{i=1}^{m}(1+j(i)) \\
& \times \exp \left\{( n - 1 ) \left[G\left(t, T, r_{t}\right)\right.\right. \\
& \left.+\int_{t}^{T} \sigma_{r}(u) m(u, T) d B(u)\right] \\
& \left.+n \int_{t}^{T}(-\lambda \theta) d s-\frac{n}{2} \sigma_{Y}^{2}\right\} \cdot e^{n Y} \\
& =S_{t}^{n} \prod_{i=1}^{m}(1+j(i)) \\
& \times \exp \left[(n-1) G\left(t, T, r_{t}\right)\right. \\
& \left.+n \int_{t}^{T}(-\lambda \theta) d s-\frac{n}{2} \sigma_{Y}^{2}\right] \cdot e^{(n-1) X+n Y},
\end{aligned}
$$

$S_{T}^{n}>K$

$$
\begin{aligned}
& \Longrightarrow S_{t}^{n} \prod_{i=1}^{m}(1+j(i)) \\
& \times \exp \left\{n \cdot\left[\int_{t}^{T} r(s) d s+\int_{t}^{T}(-\lambda \theta) d s-\frac{\sigma_{Y}^{2}}{2}\right]\right\} \\
& \cdot e^{n Y}>K \\
& \Longrightarrow S_{t} \cdot \sqrt[n]{\prod_{i=1}^{m}(1+j(i))} \\
& \quad \times \exp \left[\int_{t}^{T} r(s) d s+\int_{t}^{T}(-\lambda \theta) d s-\frac{\sigma_{Y}^{2}}{2}\right] \cdot e^{Y}>\sqrt[n]{K}
\end{aligned}
$$




$$
\begin{gathered}
\Longrightarrow \exp \left[\int_{t}^{T} \sigma_{r}(u) m(u, T) d B(u)+G\left(t, T, r_{t}\right)\right. \\
\left.\quad+\int_{t}^{T}(-\lambda \theta) d s-\frac{\sigma_{Y}^{2}}{2}\right] \cdot e^{Y} \\
>\frac{\sqrt[n]{K}}{S_{t} \cdot \sqrt[n]{\prod_{i=1}^{m}(1+j(i))}} \\
\Longrightarrow G\left(t, T, r_{t}\right)+\int_{t}^{T}(-\lambda \theta) d s-\frac{\sigma_{Y}^{2}}{2}+X+Y \\
>\ln \left[\frac{\sqrt[n]{K}}{\left.S_{t} \cdot \sqrt[n]{\prod_{i=1}^{m}(1+j(i))}\right]}\right] \\
\Longrightarrow X+Y>\ln \left[\frac{\sqrt[n]{K}}{S_{t} \cdot \sqrt[n]{\prod_{i=1}^{m}(1+j(i))}}\right] \\
-G\left(t, T, r_{t}\right)-\int_{t}^{T}(-\lambda \theta) d s+\frac{\sigma_{Y}^{2}}{2}
\end{gathered}
$$

which means

$$
\begin{aligned}
& \chi_{S_{T}^{n}>K} \\
& \quad=\chi_{X+Y>\ln \left[\sqrt[n]{K} / S_{t} \cdot \sqrt[n]{\left.\prod_{i=1}^{m}(1+j(i))\right]}-G\left(t, T, r_{t}\right)-\int_{t}^{T}(-\lambda \theta) d s+\sigma_{Y}^{2} / 2\right.}
\end{aligned}
$$

then we obtain

$$
\begin{aligned}
& I_{c}=E[ S_{t}^{n} \prod_{i=1}^{m}(1+j(i)) \\
& \times \exp \left[(n-1) G\left(t, T, r_{t}\right)+n \int_{t}^{T}(-\lambda \theta) d s-\frac{n}{2} \sigma_{Y}^{2}\right] \\
& \cdot e^{(n-1) X+n Y} \\
& \cdot \chi_{\left.X+Y>\ln \left[\sqrt[n]{K} / S_{t} \cdot \sqrt[n]{\prod_{i=1}^{m}(1+j(i))}\right]-G\left(t, T, r_{t}\right)-\int_{t}^{T}(-\lambda \theta) d s+\sigma_{Y}^{2} / 2\right]} \\
&=S_{t}^{n} \prod_{i=1}^{m}(1+j(i)) \\
& \quad \times \exp \left[(n-1) G\left(t, T, r_{t}\right)+n \int_{t}^{T}(-\lambda \theta) d s-\frac{n}{2} \sigma_{Y}^{2}\right] \\
& \cdot E\left[e^{(n-1) X+n Y}\right. \\
&\left.\quad \cdot \chi_{X+Y>\ln \left[\sqrt[n]{K} / S_{t} \cdot \sqrt[n]{\left.\prod_{i=1}^{m}(1+j(i))\right]}-G\left(t, T, r_{t}\right)-\int_{t}^{T}(-\lambda \theta) d s+\sigma_{Y}^{2} / 2\right.}\right] \\
&=S_{t}^{n} \prod_{i=1}^{m}(1+j(i))
\end{aligned}
$$

$$
\begin{aligned}
& \times \exp \left[(n-1) G\left(t, T, r_{t}\right)+n \int_{t}^{T}(-\lambda \theta) d s-\frac{n}{2} \sigma_{Y}^{2}\right] \\
\cdot & \exp \left(\frac{(n-1)^{2} \sigma_{X}^{2}+n^{2} \sigma_{Y}^{2}}{2}\right) \cdot N\left(d_{1}\right) \\
= & S_{t}^{n} \prod_{i=1}^{m}(1+j(i)) \exp \left(s_{1}\right) \cdot N\left(d_{1}\right), \\
& I I_{c}=E\left[\exp \left(-\int_{t}^{T} r(s) d s\right) K \chi_{S_{T}^{n} \geq K}\right], \\
\exp \left(-\int_{t}^{T} r(s) d s\right) & \exp \left(-G\left(t, T, r_{t}\right)-\int_{t}^{T} \sigma_{r}(u) m(u, T) d B^{Q}(u)\right) \\
= & \exp \left(-G\left(t, T, r_{t}\right)\right) \cdot \exp (-X) .
\end{aligned}
$$

In the similar way, we have

$$
\begin{gathered}
E\left[\exp \left(-\int_{t}^{T} r(s) d s\right) K \chi_{S_{T}^{n} \geq K}\right] \\
=K \cdot \exp \left(-G\left(t, T, r_{t}\right)\right) \\
\cdot E[\exp (-X)
\end{gathered}
$$

$\times \chi_{\left.X+Y>\ln \left[\sqrt[n]{K} / S_{t} \cdot \sqrt[n]{\prod_{i=1}^{m}(1+j(i))}\right]-G\left(t, T, r_{t}\right)-\int_{t}^{T}(-\lambda \theta) d s+\sigma_{Y}^{2} / 2\right]}$

$$
=K \exp \left(\frac{\sigma_{X}^{2}}{2}-G\left(t, T, r_{t}\right)\right) \cdot N\left(d_{2}\right) \text {. }
$$

Theorem 6. The formula of the first kind of put power option with exercise price $K$ at exercise date $T$ is

$$
\begin{aligned}
& P\left(t, r_{t}, S_{t}, K\right) \\
& \quad=\sum_{m=0}^{\infty}\left[\exp (-\lambda(T-t)) \frac{(\lambda(T-t))^{m}}{m !}\left(I_{p}-I I_{p}\right)\right],
\end{aligned}
$$

where

$$
\begin{gathered}
I_{p}=K \exp \left[\frac{\sigma_{X}^{2}}{2}-G\left(t, T, r_{t}\right)\right] \cdot N\left(-d_{2}\right), \\
I I_{p}=S_{t}^{n} \prod_{i=1}^{m}(1+j(i)) \exp \left(s_{1}\right) \cdot N\left(-d_{1}\right),
\end{gathered}
$$

Proof. Similar to the proof procedure in the first kind of call power option, we have

$$
I_{p}=E\left[\exp \left(-\int_{t}^{T} r(s) d s\right) K \chi_{S_{T}^{n}<K}\right] .
$$


6

Abstract and Applied Analysis

Since

$S_{T}^{n}<K$

$$
\begin{aligned}
\Longrightarrow & S_{t}^{n} \prod_{i=1}^{m}(1+j(i)) \\
& \times \exp \left\{n \cdot\left[\int_{t}^{T} r(s) d s+\int_{t}^{T}(-\lambda \theta) d s-\frac{\sigma_{Y}^{2}}{2}\right]\right\} \\
& \cdot e^{n Y}<K
\end{aligned}
$$$$
\Longrightarrow S_{t} \cdot \sqrt[n]{\prod_{i=1}^{m}(1+j(i))}
$$$$
\times \exp \left[\int_{t}^{T} r(s) d s+\int_{t}^{T}(-\lambda \theta) d s-\frac{\sigma_{Y}^{2}}{2}\right] \cdot e^{Y}<\sqrt[n]{K}
$$$$
\Longrightarrow \exp \left[\int_{t}^{T} \sigma_{r}(u) m(u, T) d B(u)+G\left(t, T, r_{t}\right)\right.
$$$$
\left.+\int_{t}^{T}(-\lambda \theta) d s-\frac{\sigma_{Y}^{2}}{2}\right] \cdot e^{Y}
$$$$
<\frac{\sqrt[n]{K}}{S_{t} \cdot \sqrt[n]{\prod_{i=1}^{m}(1+j(i))}}
$$

$\Longrightarrow G\left(t, T, r_{t}\right)+\int_{t}^{T}(-\lambda \theta) d s-\frac{\sigma_{Y}^{2}}{2}+X+Y$

$$
\begin{aligned}
& <\ln \left[\frac{\sqrt[n]{K}}{S_{t} \cdot \sqrt[n]{\prod_{i=1}^{m}(1+j(i))}}\right] \\
& \Longrightarrow X+Y<\ln \left[\frac{\sqrt[n]{K}}{S_{t} \cdot \sqrt[n]{\prod_{i=1}^{m}(1+j(i))}}\right]-G\left(t, T, r_{t}\right) \\
& -\int_{t}^{T}(-\lambda \theta) d s+\frac{\sigma_{Y}^{2}}{2},
\end{aligned}
$$

$\chi_{S_{T}^{n}<K}$

$$
\begin{aligned}
& =\chi_{-X-Y>\ln \left[S_{t} \cdot \sqrt[n]{\prod_{i=1}^{m}(1+j(i))} / \sqrt[n]{K}\right]+G\left(t, T, r_{t}\right)+\int_{t}^{T}(-\lambda \theta) d s-\sigma_{Y}^{2} / 2} \\
& \exp \left(-\int_{t}^{T} r(s) d s\right) \\
& =\exp \left(-G\left(t, T, r_{t}\right)-\int_{t}^{T} \sigma_{r}(u) m(u, T) d B^{Q}(u)\right) \\
& =\exp \left(-G\left(t, T, r_{t}\right)\right) \cdot \exp (-X)
\end{aligned}
$$

we obtain

$$
\begin{aligned}
& E\left[\exp \left(-\int_{t}^{T} r(s) d s\right) K \chi_{S_{T}^{n}<K}\right] \\
& =K \cdot \exp \left(-G\left(t, T, r_{t}\right)\right)
\end{aligned}
$$

$$
\begin{aligned}
& \cdot E[ \exp (-X) \\
&\left.\times \chi_{-X-Y>\ln \left[S_{t} \cdot \sqrt[n]{\prod_{i=1}^{m}(1+j(i))} / \sqrt[n]{K}\right]+G\left(t, T, r_{t}\right)+\int_{t}^{T}(-\lambda \theta) d s-\sigma_{Y}^{2} / 2}\right] \\
&=K \exp \left(\frac{\sigma_{X}^{2}}{2}-G\left(t, T, r_{t}\right)\right) \cdot N\left(-d_{2}\right) .
\end{aligned}
$$

Also

$$
I I_{p}=E\left[\exp \left(-\int_{t}^{T} r(s) d s\right) S_{T}^{n} \chi_{S_{T}^{n}<K}\right]
$$

Since

$$
\exp \left[-\int_{t}^{T} r(s) d s\right] S_{T}^{n}
$$$$
=\exp \left[-\int_{t}^{T} r(s) d s\right] S_{t}^{n} \prod_{i=1}^{m}(1+j(i))
$$

$$
\begin{gathered}
\times \exp \left[n \int_{t}^{T}\left(r(s)-\lambda \theta-H \sigma^{2}(s) s^{2 H-1}\right) d s\right. \\
\left.+n \int_{t}^{T} \sigma(s) d B_{H}(s)\right]
\end{gathered}
$$$$
=S_{t}^{n} \prod_{i=1}^{m}(1+j(i))
$$$$
\times \exp \left[(n-1) \int_{t}^{T} r(s) d s\right.
$$$$
\left.+n \int_{t}^{T}(-\lambda \theta) d s-\frac{n}{2} \sigma_{Y}^{2}\right] \cdot e^{n Y}
$$$$
=S_{t}^{n} \prod_{i=1}^{m}(1+j(i))
$$$$
\times \exp \left\{(n-1)\left[G\left(t, T, r_{t}\right)+\int_{t}^{T} \sigma_{r}(u) m(u, T) d B(u)\right]\right.
$$$$
\left.+n \int_{t}^{T}(-\lambda \theta) d s-\frac{n}{2} \sigma_{Y}^{2}\right\} \cdot e^{n Y}
$$$$
=S_{t}^{n} \prod_{i=1}^{m}(1+j(i))
$$

$$
\times \exp \left[(n-1) G\left(t, T, r_{t}\right)+n \int_{t}^{T}(-\lambda \theta) d s-\frac{n}{2} \sigma_{Y}^{2}\right]
$$$$
\cdot e^{(n-1) X+n Y}
$$

$S_{T}^{n}<K$ 


$$
\begin{aligned}
& \Longrightarrow S_{t}^{n} \prod_{i=1}^{m}(1+j(i)) \\
& \times \exp \left\{n \cdot\left[\int_{t}^{T} r(s) d s+\int_{t}^{T}(-\lambda \theta) d s-\frac{\sigma_{Y}^{2}}{2}\right]\right\} \\
& \cdot e^{n Y}<K \\
& \Longrightarrow S_{t} \cdot \sqrt[n]{\prod_{i=1}^{m}(1+j(i))} \\
& \times \exp \left[\int_{t}^{T} r(s) d s+\int_{t}^{T}(-\lambda \theta) d s-\frac{\sigma_{Y}^{2}}{2}\right] \cdot e^{Y}<\sqrt[n]{K} \\
& \Longrightarrow \exp \left[\int_{t}^{T} \sigma_{r}(u) m(u, T) d B(u)+G\left(t, T, r_{t}\right)\right. \\
& \left.+\int_{t}^{T}(-\lambda \theta) d s-\frac{\sigma_{Y}^{2}}{2}\right] \cdot e^{Y} \\
& <\frac{\sqrt[n]{K}}{S_{t} \cdot \sqrt[n]{\prod_{i=1}^{m}(1+j(i))}} \\
& \Longrightarrow G\left(t, T, r_{t}\right)+\int_{t}^{T}(-\lambda \theta) d s-\frac{\sigma_{Y}^{2}}{2}+X+Y \\
& <\ln \left[\frac{\sqrt[n]{K}}{S_{t} \cdot \sqrt[n]{\prod_{i=1}^{m}(1+j(i))}}\right] \\
& \Longrightarrow X+Y<\ln \left[\frac{\sqrt[n]{K}}{S_{t} \cdot \sqrt[n]{\prod_{i=1}^{m}(1+j(i))}}\right]-G\left(t, T, r_{t}\right) \\
& -\int_{t}^{T}(-\lambda \theta) d s+\frac{\sigma_{Y}^{2}}{2},
\end{aligned}
$$

$\chi_{S_{T}^{n}<K}$

$$
=\chi_{-X-Y>\ln \left[S_{t} \cdot \sqrt[n]{\prod_{i=1}^{m}(1+j(i))} / \sqrt[n]{K}\right]+G\left(t, T, r_{t}\right)+\int_{t}^{T}(-\lambda \theta) d s-\sigma_{Y}^{2} / 2}
$$

$$
\begin{aligned}
& \cdot e^{(n-1) X+n Y}
\end{aligned}
$$

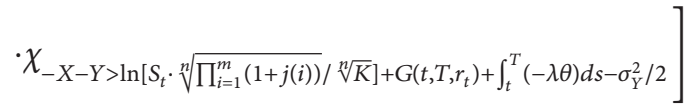

$$
\begin{aligned}
& =S_{t}^{n} \prod_{i=1}^{m}(1+j(i)) \\
& \times \exp \left[(n-1) G\left(t, T, r_{t}\right)+n \int_{t}^{T}(-\lambda \theta) d s-\frac{n}{2} \sigma_{Y}^{2}\right] \\
& \cdot E\left[e^{(n-1) X+n Y}\right.
\end{aligned}
$$

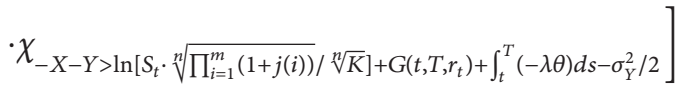

$$
\begin{aligned}
& =S_{t}^{n} \prod_{i=1}^{m}(1+j(i)) \\
& \times \exp \left[(n-1) G\left(t, T, r_{t}\right)+n \int_{t}^{T}(-\lambda \theta) d s-\frac{n}{2} \sigma_{Y}^{2}\right] \\
& \cdot \exp \left(\frac{(n-1)^{2} \sigma_{X}^{2}+n^{2} \sigma_{Y}^{2}}{2}\right) \\
& \cdot N\left[\left(-\ln \left[\frac{S_{t} \cdot \sqrt[n]{\prod_{i=1}^{m}(1+j(i))}}{\sqrt[n]{K}}\right]-G\left(t, T, r_{t}\right)\right.\right. \\
& \left.+\int_{t}^{T}(\lambda \theta) d s-(n-1) \sigma_{X}^{2}-\frac{(2 n-1)}{2} \sigma_{Y}^{2}\right) \\
& \left.\times\left(\sqrt{\sigma_{X}^{2}+\sigma_{Y}^{2}}\right)^{-1}\right] \\
& =S_{t}^{n} \prod_{i=1}^{m}(1+j(i)) \\
& \times \exp \left[\frac{(n-1)^{2}}{2} \sigma_{X}^{2}+\frac{n^{2}-n}{2} \sigma_{Y}^{2}+(n-1) G\left(t, T, r_{t}\right)\right. \\
& \left.-n \int_{t}^{T} \lambda \theta d s\right] \cdot N\left(-d_{1}\right) .
\end{aligned}
$$

we obtain

$$
\begin{array}{ll}
E\left[\exp \left(-\int_{t}^{T} r(s) d s\right) S_{T}^{n} \chi_{S_{T}^{n}<K}\right] & \begin{array}{l}
\text { Theorem } 7 . \text { The formula of the second kind call power option } \\
\text { with exercise price } K, \text { exercise date } T \text { is }
\end{array} \\
=E\left[S_{t}^{n} \prod_{i=1}^{m}(1+j(i))\right. & C\left(t, r_{t}, S_{t}, K\right) \\
\quad \times \exp \left[(n-1) G\left(t, T, r_{t}\right)+n \int_{t}^{T}(-\lambda \theta) d s-\frac{n}{2} \sigma_{Y}^{2}\right] & =\sum_{m=0}^{\infty}\left[\exp (-\lambda(T-t)) \frac{(\lambda(T-t))^{m}}{m !}\left(I I I_{c}-I V_{c}\right)\right],
\end{array}
$$

\subsection{Pricing of the Second Kind Power Option}


where

$I I I_{c}$

$$
\begin{aligned}
&=S_{t}^{n} \prod_{i=1}^{m}(1+j(i)) \\
& \times \exp \left[\frac{(n-1)^{2}}{2} \sigma_{X}^{2}+\frac{n^{2}-n}{2} \sigma_{Y}^{2}\right. \\
&\left.\left.+(n-1) G\left(t, T, r_{t}\right)-n\right]_{t}^{T} \lambda \theta d s\right] \cdot N\left(d_{3}\right), \\
& I V_{c}= K \exp \left[\frac{\sigma_{X}^{2}}{2}-G\left(t, T, r_{t}\right)\right] \cdot N\left(d_{4}\right), \\
& N\left(d_{3}\right) \quad \quad\left(\ln \left[S_{t} \cdot \frac{\prod_{i=1}^{m}(1+j(i))}{K}\right]+G\left(t, T, r_{t}\right)\right. \\
&=N\left(\int_{t}^{T} \lambda \theta d s+(n-1) \sigma_{X}^{2}+\frac{2 n-1}{2} \sigma_{Y}^{2}\right) \\
&\left.\quad \times\left(\sqrt{\sigma_{X}^{2}+\sigma_{Y}^{2}}\right)^{-1}\right),
\end{aligned}
$$

$N\left(d_{4}\right)$

$$
\begin{aligned}
=N( & \left(\ln \left[S_{t} \cdot \frac{\prod_{i=1}^{m}(1+j(i))}{K}\right]+G\left(t, T, r_{t}\right)\right. \\
& \left.\left.-\int_{t}^{T} \lambda \theta d s-\sigma_{X}^{2}-\frac{\sigma_{Y}^{2}}{2}\right)\left(\sqrt{\sigma_{X}^{2}+\sigma_{Y}^{2}}\right)^{-1}\right) .
\end{aligned}
$$

Proof. Similarly, we have

$$
I I I_{c}=E\left[\exp \left(-\int_{t}^{T} r(s) d s\right) S_{T}^{n} \chi_{S_{T} \geq K}\right]
$$

Since

$$
\begin{aligned}
& \exp \left[-\int_{t}^{T} r(s) d s\right] S_{T}^{n} \\
& =\exp \left[-\int_{t}^{T} r(s) d s\right] S_{t}^{n} \prod_{i=1}^{m}(1+j(i)) \\
& \times \exp \left[n \int_{t}^{T}\left(r(s)-\lambda \theta-H \sigma^{2}(s) s^{2 H-1}\right) d s\right. \\
& \left.+n \int_{t}^{T} \sigma(s) d B_{H}(s)\right]
\end{aligned}
$$

$$
\begin{aligned}
= & S_{t}^{n} \prod_{i=1}^{m}(1+j(i)) \\
& \times \exp \left[(n-1) \int_{t}^{T} r(s) d s+n \int_{t}^{T}(-\lambda \theta) d s-\frac{n}{2} \sigma_{Y}^{2}\right] \cdot e^{n Y} \\
= & S_{t}^{n} \prod_{i=1}^{m}(1+j(i)) \\
& \times \exp \left\{(n-1)\left[G\left(t, T, r_{t}\right)+\int_{t}^{T} \sigma_{r}(u) m(u, T) d B(u)\right]\right. \\
& \left.\quad+n \int_{t}^{T}(-\lambda \theta) d s-\frac{n}{2} \sigma_{Y}^{2}\right\} \cdot e^{n Y} \\
= & S_{t}^{n} \prod_{i=1}^{m}(1+j(i)) \\
& \times \exp \left[(n-1) G\left(t, T, r_{t}\right)+n \int_{t}^{T}(-\lambda \theta) d s-\frac{n}{2} \sigma_{Y}^{2}\right] \\
& \cdot e^{(n-1) X+n Y},
\end{aligned}
$$$$
S_{T} \geq K
$$$$
\Longrightarrow S_{t} \prod_{i=1}^{m}(1+j(i))
$$$$
\times \exp \left[\int_{t}^{T} r(s) d s+\int_{t}^{T}(-\lambda \theta) d s-\frac{\sigma_{Y}^{2}}{2}\right] \cdot e^{Y} \geq K
$$$$
\Longrightarrow \exp \left[\int_{t}^{T} \sigma_{r}(u) m(u, T) d B(u)+G\left(t, T, r_{t}\right)\right.
$$$$
\left.+\int_{t}^{T}(-\lambda \theta) d s-\frac{\sigma_{Y}^{2}}{2}\right] \cdot e^{Y}
$$$$
>\frac{K}{S_{t} \cdot \prod_{i=1}^{m}(1+j(i))}
$$$$
\Longrightarrow G\left(t, T, r_{t}\right)+\int_{t}^{T}(-\lambda \theta) d s-\frac{\sigma_{Y}^{2}}{2}+X+Y
$$$$
\geq \ln \left[\frac{K}{S_{t} \cdot \prod_{i=1}^{m}(1+j(i))}\right]
$$$$
\Longrightarrow X+Y \geq \ln \left[\frac{K}{S_{t} \cdot \prod_{i=1}^{m}(1+j(i))}\right]
$$$$
-G\left(t, T, r_{t}\right)-\int_{t}^{T}(-\lambda \theta) d s+\frac{\sigma_{Y}^{2}}{2},
$$

$\chi_{S_{T} \geq K}=\chi_{X+Y \geq \ln \left[K / S_{t} \cdot \prod_{i=1}^{m}(1+j(i))\right]-G\left(t, T, r_{t}\right)-\int_{t}^{T}(-\lambda \theta) d s+\sigma_{Y}^{2} / 2}$,

we obtain

$$
\begin{aligned}
& E\left[\exp \left(-\int_{t}^{T} r(s) d s\right) S_{T}^{n} \chi_{S_{T} \geq K}\right] \\
& =E\left[S_{t}^{n} \prod_{i=1}^{m}(1+j(i))\right.
\end{aligned}
$$




$$
\begin{aligned}
& \times \exp \left[(n-1) G\left(t, T, r_{t}\right)+n \int_{t}^{T}(-\lambda \theta) d s-\frac{n}{2} \sigma_{Y}^{2}\right] \\
& \cdot e^{(n-1) X+n Y} \\
& \left.\cdot \chi_{X+Y \geq \ln \left[K / S_{t} \cdot \prod_{i=1}^{m}(1+j(i))\right]-G\left(t, T, r_{t}\right)-\int_{t}^{T}(-\lambda \theta) d s+\sigma_{Y}^{2} / 2}\right] \\
& =S_{t}^{n} \prod_{i=1}^{m}(1+j(i)) \\
& \times \exp \left[(n-1) G\left(t, T, r_{t}\right)+n \int_{t}^{T}(-\lambda \theta) d s-\frac{n}{2} \sigma_{Y}^{2}\right] \\
& \cdot E\left[e^{(n-1) X+n Y}\right. \\
& \left.\cdot \chi_{X+Y \geq \ln \left[K / S_{t} \cdot \prod_{i=1}^{m}(1+j(i))\right]-G\left(t, T, r_{t}\right)-\int_{t}^{T}(-\lambda \theta) d s+\sigma_{Y}^{2} / 2}\right] \\
& =S_{t}^{n} \prod_{i=1}^{m}(1+j(i)) \\
& \times \exp \left[(n-1) G\left(t, T, r_{t}\right)+n \int_{t}^{T}(-\lambda \theta) d s-\frac{n}{2} \sigma_{Y}^{2}\right] \\
& \cdot \exp \left(\frac{(n-1)^{2} \sigma_{X}^{2}+n^{2} \sigma_{Y}^{2}}{2}\right) \\
& \cdot N\left[\left(\ln \left[\frac{S_{t} \cdot \prod_{i=1}^{m}(1+j(i))}{K}\right]+G\left(t, T, r_{t}\right)\right.\right. \\
& \left.-\int_{t}^{T}(\lambda \theta) d s+(n-1) \sigma_{X}^{2}+\frac{(2 n-1)}{2} \sigma_{Y}^{2}\right) \\
& \left.\times\left(\sqrt{\sigma_{X}^{2}+\sigma_{Y}^{2}}\right)^{-1}\right] \\
& =S_{t}^{n} \prod_{i=1}^{m}(1+j(i)) \\
& \times \exp \left[\frac{(n-1)^{2}}{2} \sigma_{X}^{2}+\frac{n^{2}-n}{2} \sigma_{Y}^{2}+(n-1) G\left(t, T, r_{t}\right)\right. \\
& \left.-n \int_{t}^{T} \lambda \theta d s\right] \cdot N\left(d_{3}\right) .
\end{aligned}
$$

Also,

$$
I V_{c}=E\left[\exp \left(-\int_{t}^{T} r(s) d s\right) K \chi_{S_{T} \geq K}\right]
$$

where

$$
\begin{aligned}
& \chi_{S_{T} \geq K} \\
& \quad=\chi_{X+Y \geq \ln \left[K / S_{t} \prod_{i=1}^{m}(1+j(i))\right]-G\left(t, T, r_{t}\right)-\int_{t}^{T}(-\lambda \theta) d s+\sigma_{Y}^{2} / 2}, \\
& \exp \left(-\int_{t}^{T} r(s) d s\right) \\
& \quad=\exp \left(-G\left(t, T, r_{t}\right)-\int_{t}^{T} \sigma_{r}(u) m(u, T) d B^{Q}(u)\right) \\
& \quad=\exp \left(-G\left(t, T, r_{t}\right)\right) \cdot \exp (-X) .
\end{aligned}
$$

In the similar way, we have

$$
\begin{aligned}
& E\left[\exp \left(-\int_{t}^{T} r(s) d s\right) K \chi_{S_{T} \geq K}\right] \\
&=K \cdot \exp \left(-G\left(t, T, r_{t}\right)\right) \\
& \quad \cdot E[\exp (-X) \\
&\left.\quad \times \chi_{X+Y \geq \ln \left[K / S_{t} \cdot \prod_{i=1}^{m}(1+j(i))\right]-G\left(t, T, r_{t}\right)-\int_{t}^{T}(-\lambda \theta) d s+\sigma_{Y}^{2} / 2}\right] \\
&=K \exp \left(\frac{\sigma_{X}^{2}}{2}-G\left(t, T, r_{t}\right)\right) \cdot N\left(d_{4}\right) .
\end{aligned}
$$

Theorem 8. The formula of the second kind put power option with exercise price $K$, exercise date $T$ is

$$
\begin{aligned}
P & \left(t, r_{t}, S_{t}, K\right) \\
\quad & \sum_{m=0}^{\infty}\left[\exp (-\lambda(T-t)) \frac{(\lambda(T-t))^{m}}{m !}\left(I I I_{p}-I V_{p}\right)\right],
\end{aligned}
$$

where

$$
\begin{gathered}
I I I_{p}=K \exp \left[\frac{\sigma_{X}^{2}}{2}-G\left(t, T, r_{t}\right)\right] \cdot N\left(-d_{4}\right), \\
I V_{p}=S_{t}^{n} \prod_{i=1}^{m}(1+j(i)) \\
\quad \times \exp \left[\frac{(n-1)^{2}}{2} \sigma_{X}^{2}+\frac{n^{2}-n}{2} \sigma_{Y}^{2}\right.
\end{gathered}
$$




$$
\begin{gathered}
\left.+(n-1) G\left(t, T, r_{t}\right)-n \int_{t}^{T} \lambda \theta d s\right] \cdot N\left(-d_{3}\right), \\
N\left(-d_{4}\right)=N\left(\left(-\ln \left[S_{t} \cdot \frac{\prod_{i=1}^{m}(1+j(i))}{K}\right]-G\left(t, T, r_{t}\right)\right.\right. \\
\left.+\int_{t}^{T} \lambda \theta d s+\sigma_{X}^{2}+\frac{\sigma_{Y}^{2}}{2}\right) \\
\left.\times\left(\sqrt{\sigma_{X}^{2}+\sigma_{Y}^{2}}\right)^{-1}\right), \\
N\left(-d_{3}\right)=N\left(\left(-\ln \left[S_{t} \cdot \frac{\prod_{i=1}^{m}(1+j(i))}{K}\right]-G\left(t, T, r_{t}\right)\right.\right. \\
\left.+\int_{t}^{T} \lambda \theta d s-(n-1) \sigma_{X}^{2}-\frac{2 n-1}{2} \sigma_{Y}^{2}\right) \\
\left.\times\left(\sqrt{\sigma_{X}^{2}+\sigma_{Y}^{2}}\right)^{-1}\right) .
\end{gathered}
$$

Proof. Similarly, we have

$$
I I I_{p}=E\left[\exp \left(-\int_{t}^{T} r(s) d s\right) K \chi_{S_{T}<K}\right] .
$$

$$
\begin{aligned}
& \text { Since } \\
& \begin{array}{l}
\chi_{S_{T}<K} ! \\
\quad=\chi_{-X-Y>\ln \left[S_{t} \cdot \prod_{i=1}^{m}(1+j(i)) / \sqrt[n]{K}\right]+G\left(t, T, r_{t}\right)+\int_{t}^{T}(-\lambda \theta) d s-\sigma_{Y}^{2} / 2}, \\
\quad \exp \left(-\int_{t}^{T} r(s) d s\right) \\
\quad=\exp \left(-G\left(t, T, r_{t}\right)-\int_{t}^{T} \sigma_{r}(u) m(u, T) d B^{Q}(u)\right) \\
\quad=\exp \left(-G\left(t, T, r_{t}\right)\right) \cdot \exp (-X),
\end{array}
\end{aligned}
$$

we obtain

$$
\begin{aligned}
& E\left[\exp \left(-\int_{t}^{T} r(s) d s\right) K \chi_{S_{T}<K}\right] \\
& =K \cdot \exp \left(-G\left(t, T, r_{t}\right)\right) \\
& \quad \cdot E[\exp (-X) \\
& \left.\quad \times \chi_{\left.-X-Y>\ln \left[S_{t} \cdot \prod_{i=1}^{m}(1+j(i)) / K\right]+G\left(t, T, r_{t}\right)+\int_{t}^{T}(-\lambda \theta) d s-\sigma_{Y}^{2} / 2\right]}\right] \\
& =K \exp \left(\frac{\sigma_{X}^{2}}{2}-G\left(t, T, r_{t}\right)\right) \cdot N\left(-d_{4}\right) .
\end{aligned}
$$

Also,

$$
I V_{p}=E\left[\exp \left(-\int_{t}^{T} r(s) d s\right) S_{T}^{n} \chi_{S_{T}<K}\right] .
$$

Since

$$
\begin{aligned}
& \exp \left[-\int_{t}^{T} r(s) d s\right] S_{T}^{n} \\
& =\exp \left[-\int_{t}^{T} r(s) d s\right] S_{t}^{n} \prod_{i=1}^{m}(1+j(i)) \\
& \times \exp \left[n \int_{t}^{T}\left(r(s)-\lambda \theta-H \sigma^{2}(s) s^{2 H-1}\right) d s\right. \\
& \left.\quad+n \int_{t}^{T} \sigma(s) d B_{H}(s)\right] \\
& =S_{t}^{n} \prod_{i=1}^{m}(1+j(i)) \\
& \quad \times \exp \left[(n-1) \int_{t}^{T} r(s) d s+n \int_{t}^{T}(-\lambda \theta) d s-\frac{n}{2} \sigma_{Y}^{2}\right] \cdot e^{n Y} \\
& =S_{t}^{n} \prod_{i=1}^{m}(1+j(i)) \\
& \quad \times \exp \left\{(n-1)\left[G\left(t, T, r_{t}\right)+\int_{t}^{T} \sigma_{r}(u) m(u, T) d B(u)\right]\right. \\
& \left.\quad+n \int_{t}^{T}(-\lambda \theta) d s-\frac{n}{2} \sigma_{Y}^{2}\right\} \cdot e^{n Y} \\
& \quad \chi_{S_{T}<K}=\chi_{-X-Y>\ln \left[S_{t} \cdot \prod_{i=1}^{m}(1+j(i)) / K\right]+G\left(t, T, r_{t}\right)+\int_{t}^{T}(-\lambda \theta) d s-\sigma_{Y}^{2} / 2} \\
& \quad \times \quad \exp \left[(n-1) G\left(t, T, r_{t}\right)\right. \\
& \quad \prod_{i=1}^{n}(1+j(i)) \\
& \quad
\end{aligned}
$$

we obtain

$$
\begin{aligned}
& E\left[\exp \left(-\int_{t}^{T} r(s) d s\right) S_{T}^{n} \chi_{S_{T}<K}\right] \\
& =E\left[S_{t}^{n} \prod_{i=1}^{m}(1+j(i))\right. \\
& \quad \times \exp \left[(n-1) G\left(t, T, r_{t}\right)+n \int_{t}^{T}(-\lambda \theta) d s-\frac{n}{2} \sigma_{Y}^{2}\right] \\
& \quad \cdot e^{(n-1) X+n Y} \\
& \left.\quad \cdot \chi_{-X-Y>\ln \left[S_{t} \cdot \prod_{i=1}^{m}(1+j(i)) / K\right]+G\left(t, T, r_{t}\right)+\int_{t}^{T}(-\lambda \theta) d s-\sigma_{Y}^{2} / 2}\right] \\
& =S_{t}^{n} \prod_{i=1}^{m}(1+j(i))
\end{aligned}
$$




$$
\begin{aligned}
& \times \exp \left[(n-1) G\left(t, T, r_{t}\right)+n \int_{t}^{T}(-\lambda \theta) d s-\frac{n}{2} \sigma_{Y}^{2}\right] \\
& \cdot \exp \left(\frac{(n-1)^{2} \sigma_{X}^{2}+n^{2} \sigma_{Y}^{2}}{2}\right) \\
& \cdot N\left[\left(-\ln \left[\frac{S_{t} \cdot \prod_{i=1}^{m}(1+j(i))}{K}\right]-G\left(t, T, r_{t}\right)\right.\right. \\
& \left.\quad+\int_{t}^{T}(\lambda \theta) d s-(n-1) \sigma_{X}^{2}-\frac{(2 n-1)}{2} \sigma_{Y}^{2}\right) \\
& \left.\quad \times\left(\sqrt{\sigma_{X}^{2}+\sigma_{Y}^{2}}\right)^{-1}\right] \\
& =S_{t}^{n} \prod_{i=1}^{m}(1+j(i)) \\
& \times \exp \left[\frac{(n-1)^{2}}{2} \sigma_{X}^{2}+\frac{n^{2}-n}{2} \sigma_{Y}^{2}+(n-1) G\left(t, T, r_{t}\right)\right. \\
& \left.\quad-n \int_{t}^{T} \lambda \theta d s\right] \cdot N\left(-d_{3}\right) .
\end{aligned}
$$

\section{Conclusions}

In this paper, we investigate the issue of pricing the reset option in FBM model, closed-form formulas for the reset option with a single reset date, and the phenomena of delta of the reset jumps existing in the reset option during the reset date. Under the assumption that the exchange rate follows the extended Vasicek model, we obtain the closed form of the pricing formulas for two kinds of power options under fractional Brownian motion(FBM) jump-diffusion models.

\section{Conflict of Interests}

The authors declare that there is no conflict of interest regarding the publication of this paper.

\section{Acknowledgments}

Thanks are due to the referees whose meaningful suggestions are very helpful to revise the paper. This work is supported by the Fundamental Research Funds for the Central Universities (JBK140924, JBK130401).

\section{References}

[1] F. Black and M. Scholes, "The pricing of options and corporate liabilities," Journal of Political Economy, vol. 81, pp. 637-654, 1973.

[2] Y. Hu and B. Oksendal, "Fractional white noise calculus and application to finance," Infinite Dimensional Analysis, Quantum Probability and Related Topics, vol. 6, no. 1, pp. 1-32, 2003.
[3] R. J. Elliott and J. van der Hoek, "A general fractional white noise theory and applications to finance," Mathematical Finance, vol. 13, no. 2, pp. 301-330, 2003.

[4] C. Necula, "Option pricing in a fractional Brownian motion environment," Pure Mathematics, vol. 2, pp. 63-68, 2002.

[5] S. Y. Liu and X. Q. Yang, "Pricing of European contingent claim in fractional Brownian motion environment," Chinese Jounanl of Applied Probability and Statistics, vol. 20, no. 4, pp. 429-434, 2004.

[6] S. Y. Liu and X. Q. Yang, "Pricing of compound option in a fractional Brownian motion environment," Chinese Journal of Engineering Mathematics, vol. 23, no. 1, pp. 153-157, 2006.

[7] H. Xue and L. S. Wang, "Pricing of maximum or minimum option in the fractional Brownian motion environment," Chinese Journal of Engineering Mathematics, vol. 25, no. 5, pp. 843$850,2008$.

[8] R. J. Elliott and L. Chan, "Perpetual American options with fractional Brownian motion," Quantitative Finance, vol. 4, no. 2, pp. 123-128, 2004.

[9] D. H. Peng, "Pricing of perpetual American put with fractional O-U process," Acta Mathematica Scientia A, vol. 27, no. 6, pp. 1141-1147, 2007.

[10] G. H. Deng and H. Y. Lin, "Pricing American put option in a fractional black-Scholes model via compound option approximation," Advance in Systems Science and Applications, vol. 8, no. 3, pp. 447-456, 2008.

[11] X. Deng, Option Pricing under Stochastic Rate in Fractional Brownian Motion, Chongqing University, 2009. 


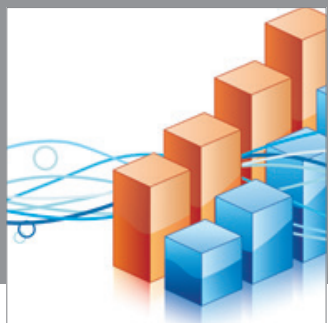

Advances in

Operations Research

mansans

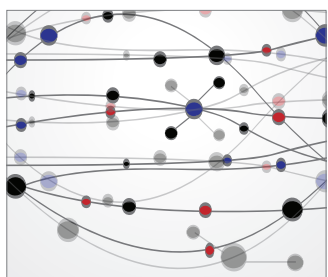

The Scientific World Journal
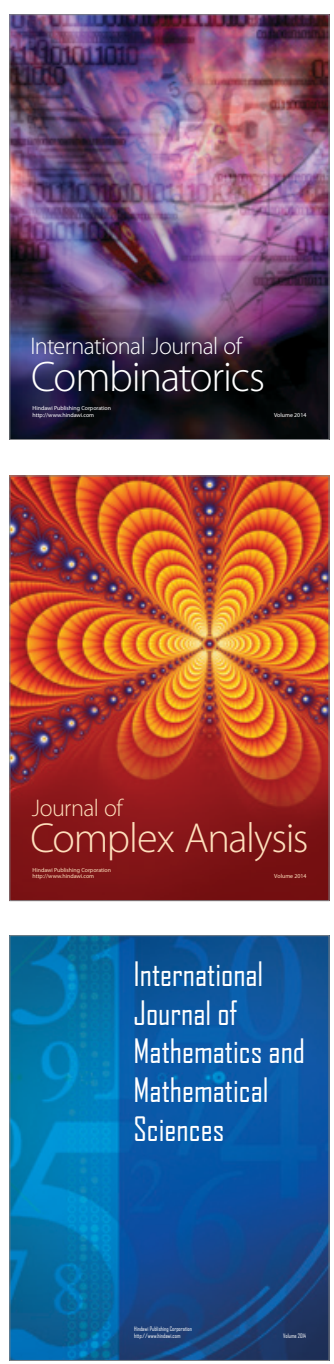
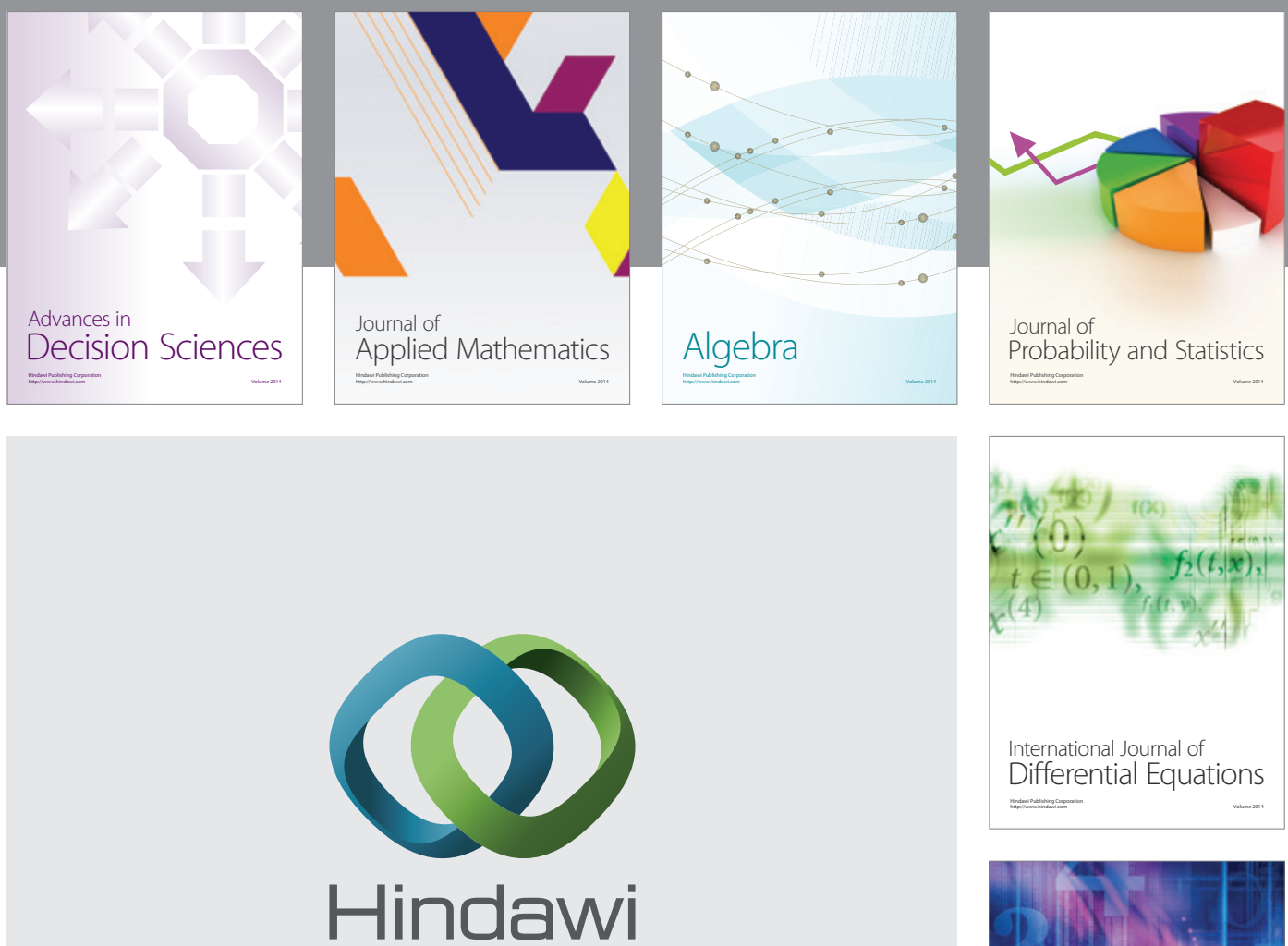

Submit your manuscripts at http://www.hindawi.com
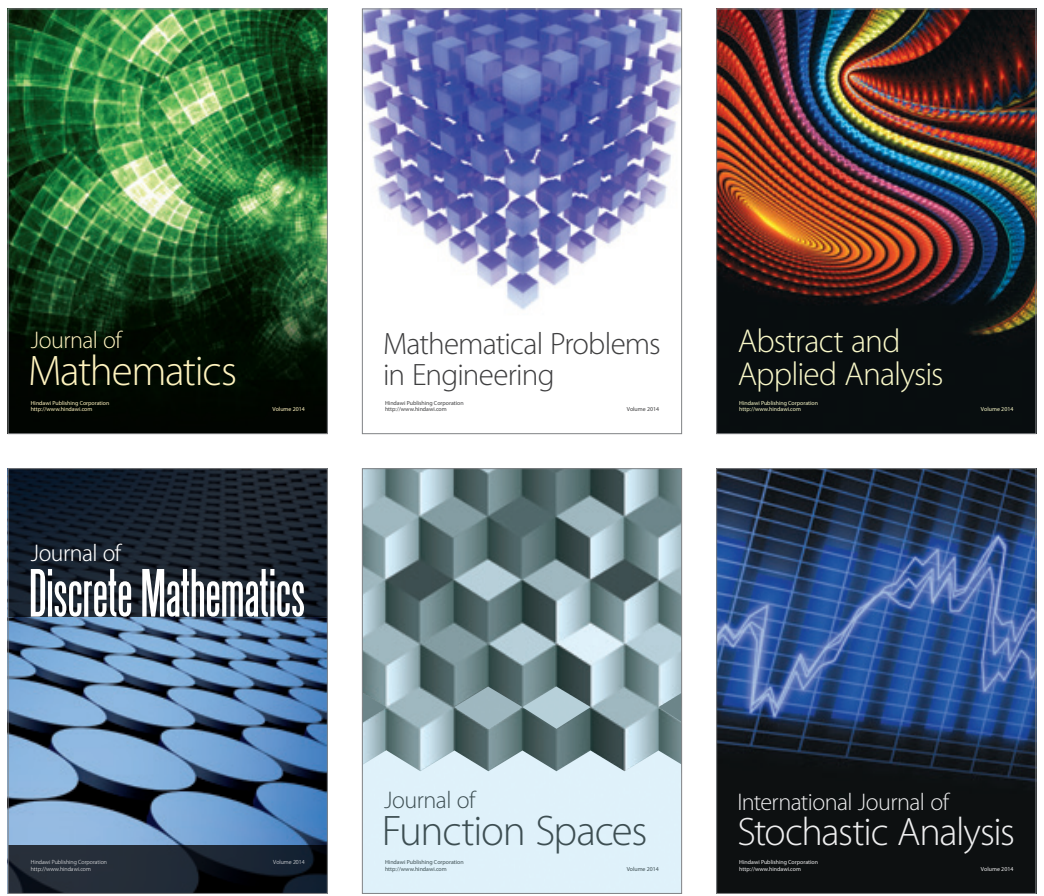

Journal of

Function Spaces

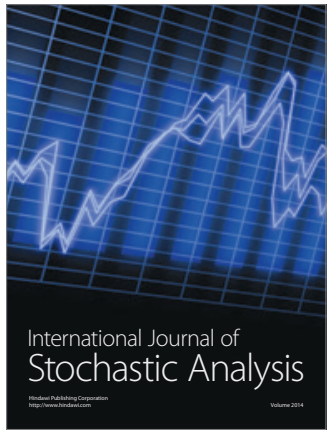

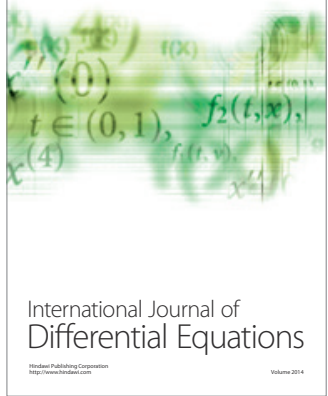
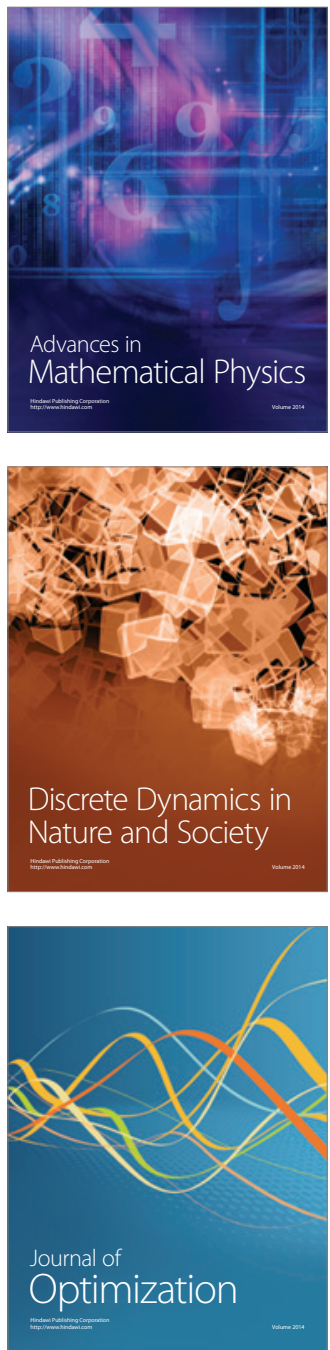